"Exzellente Redner zu ungewöhnlichen Themen wie

,Neuroökonomie' und ,Hirnforschung trifft IT' erwarten

Sie in der Eröffnungsveranstaltung ..."

Prof. Dr. med. Dr. h.c. Stefan Schwab, Erlangen

DGN-Kongresspräsident 2016

\title{
Mannheim erwartet Sie!
}

\section{$\mathrm{L}$} iebe Kolleginnen und Kollegen, ich möchte Sie ganz herzlich zum 89. Kongress der Deutschen Gesellschaft für Neurologie mit Fortbildungsakademie vom 21. bis 24 . September 2016 nach Mannheim einladen. Ein spannendes und hochaktuelles wissenschaftliches Programm wird in vier Tagen das gesamte Spektrum der Neurologie abdecken. 80 wissenschaftliche Symposien, Workshops und Minisymposien mit 580 Vorträgen und 315 Postern, dazu 23 Satellitensymposien, eine große Fachausstellung, das berufspolitische Programm im DGN-Forum und ein Nachwuchsprogramm erwarten Sie. Hinzu kommt das unabhängige Programm der DGNFortbildungsakademie mit 55 Fortbildungskursen und insgesamt 320 Vorträgen, in dem ausgewiesene Experten der deutschen Neurologie praxisnahe Themen präsentieren.

Die Eröffnungsveranstaltung - zweifelsohne bereits ein Höhepunkt des DGN-Kongresses - bestreiten zwei exzellente Redner mit außergewöhnlichen Vorträgen: Professor Christian Elger, Direktor der Klinik für Epileptologie in Bonn, wird in seinem Vortrag zur "Neuroökonomie“ faszinierende Einblicke in die Interaktion von Hirnforschung und Ökonomie geben. Was passiert in unserem Gehirn etwa wenn wir auf etwas wetten oder bieten? Professor Klaus-Robert Müller vom Institut für Softwaretechnik und Theoretische Informatik an der TU Berlin vermittelt uns einen Überblick zu „Machine Learning und Künstliche Intelligenz“. Unter dem Motto „Hirnforschung trifft IT“ blickt er für uns in die Zukunft und zeigt, wie sich diese Forschungsfelder entwickeln und intelligente Systeme unseren Lebensalltag beeinflussen und optimieren werden.

\section{Klinische Schwerpunktthemen}

$\mathrm{Zu}$ den klinischen Schwerpunktthemen gehören 2016 Schlaganfall, neurologische Intensivmedizin, Multiple Sklerose, Parkinson-Erkrankung sowie Epilepsien. Den Fokus setzen wir - bereits im Rah- men des Präsidentensymposiums (Donnerstag, 22. September, 17.00 bis $20.00 \mathrm{Uhr}$ ) - auf invasive Therapieoptionen. Im Mittelpunkt stehen wegweisende Fortschritte in Forschung und Diagnostik. Mit interessanten Fragestellungen, Forschungsergebnissen und -ansätzen werden internationale Kollegen mit ihren Vorträgen für Gesprächsstoff sorgen: Den Anfang macht Professor Jonas Frisén vom Karolinska Institutet Stockholm, der über seine Forschung zu Stammzellen und ihr therapeutisches Potenzial referiert. Professor Thomas Foltynies vom National Hospital for Neurology \& Neurosurgery in London beantwortet in seinem Vortrag über „Neurodegeneration: Transplantation fetaler Zellen - wo stehen wir?“ zukunftsweisende Fragen. Der Leiter der Klinik für Neurologie und der Poliklinik des Universitätsspitals Basel, Professor Ludwig Kappos, widmet sich in der Neuroimmunologie der Frage „Brauchen wir mehr Zelldepletion und Myeloablation?“ und geht auf die Therapieoptionen und Gefahren von Stammzelltransplantationen und selektiven monoklonalen Antikörpern bei der Multiplen Sklerose ein.

\section{Thrombektomiestudien - der Weg in die Praxis}

Professor Martin Köhrmann, stellvertretender Direktor der Universitätsklinik für Neurologie Erlangen, spricht über „Zerebrale Ischämie: je invasiver, je besser?" und beleuchtet den Quantensprung in der Schlaganfalltherapie seit letztem Jahr durch die sehr positiven Thrombektomiestudien. In diesem Jahr gehen wir der Frage nach, wie sich die positiven Ergebnisse der Thrombektomiestudien in die klinische Routine übertragen lassen. Wir diskutieren, wie die Strukturen in Deutschland angepasst werden müssen. Brauchen wir zum Beispiel eher viele interventionelle oder besser wenige gut ausgestattete Zentren? Das eine bedeutet eine flächendeckende Versorgung, das andere eine Zentralisierung mit einer speziellen Patientenlogistik. Die Thrombektomie wird von Neuroradiologen durch- 


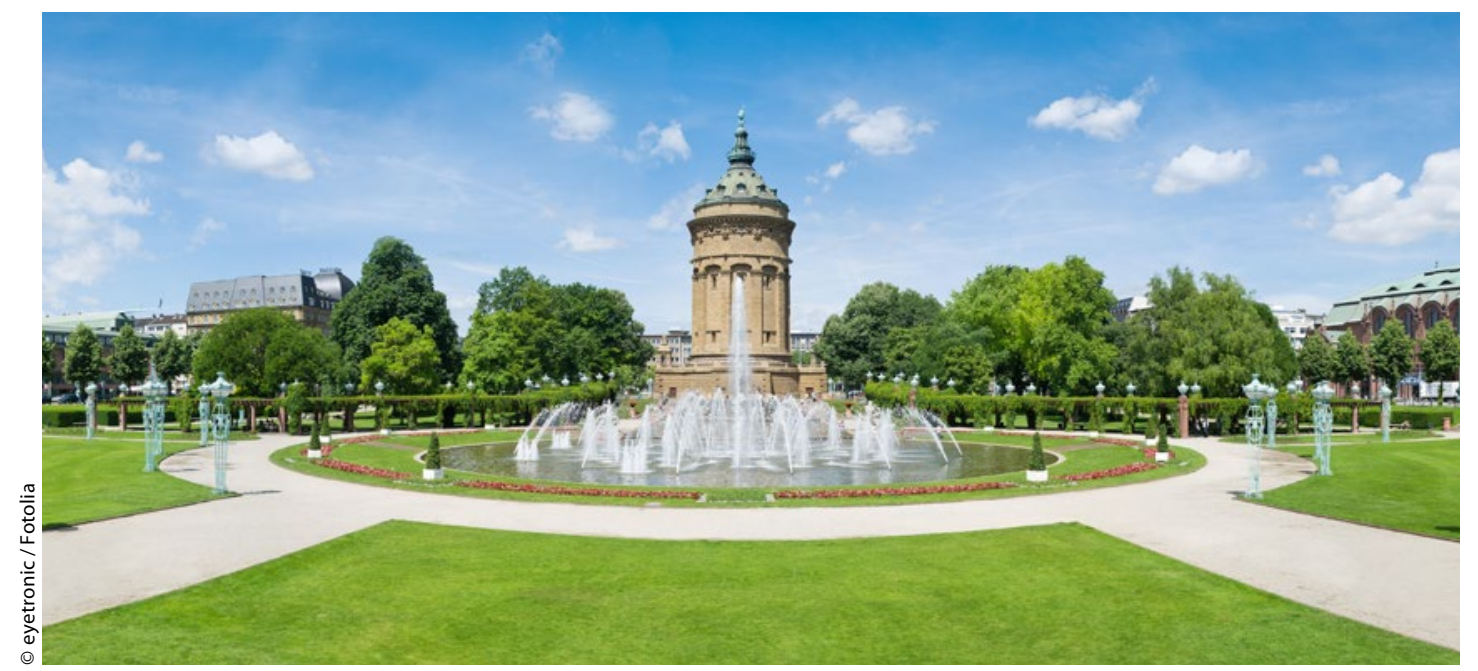

Zum 89. DGN-Kongress im September 2016 sind alle Interessierten herzlich nach Mannheim eingeladen.

geführt - und diese gibt es nicht an jeder Klinik. Wir werden überlegen, wie wir eine Rund-um-dieUhr-Versorgung der Schlaganfallpatienten gewährleisten können.

Professor Andreas Schulz-Bonhage, Leiter des Epilepsiezentrums der Universitätsklinik Freiburg, wird über die „Klinische Wertigkeit und Zukunft der Stimulationsverfahren“ in der Epilepsie referieren. Auch hier haben invasive Ableitung und Stimulationsverfahren in den letzten Jahren immens an Bedeutung gewonnen. Ein Schwerpunkt des Kongresses liegt auf der invasiven Diagnostik und Maßnahmen, die für eine Operation der Epilepsiepatienten notwendig sind. Es werden Verfahren wie Vagusstimulation und Tiefenelektroden im Rahmen der prächirurgischen Diagnostik bei der Versorgung fokaler Epilepsien vorgestellt. Als Ergänzung zu den invasiven Diagnostik- und Therapiekonzepten wird Professor Agnes Flöel, Charité Berlin, auf nicht invasive Stimulationsverfahren als wertvolles Therapieverfahren zur Behandlung von degenerativen oder neurovaskulären Erkrankungen eingehen.

\section{Hirntod und das neue Gesetz zur Diagnostik}

Das Thema des irreversiblen Hirnfunktionsausfalls ist nicht nur für Ärzte wichtig, sondern es besitzt auch gesellschaftliche Relevanz. Aufgrund negativer Schlagzeilen in den letzten Jahren ist das gesamte Feld in Bewegung: Derzeit werden die Abläufe vor und während der Organspende optimiert und die Transparenz zurecht deutlich erhöht. Wir wollen verschiedene Fragen beantworten, zum Beispiel: Gibt es Fallstricke, und wenn ja, welche? Können Fehler in speziellen Situationen auftreten und welche sind das? Wir wollen diskutieren, wo Unsicher- heiten bestehen und Kurse anbieten, die bestimmte Situationen beleuchten.

\section{Kritische historische Aufarbeitung}

Als ganz wichtigen weiteren Programmpunkt werden wir die im letzten Jahr in Düsseldorf begonnene Diskussion über die Rolle der Neurologie in der NS-Zeit fortsetzen. Das Thema hat beim letzten DGN-Kongress sehr viele Kollegen interessiert und auch emotional bewegt. In einem Symposium wird unter anderen der renommierte Medizinhistoriker und -ethiker Professor Axel Karenberg, Institut für Geschichte und Ethik der Medizin der Universität zu Köln, exemplarisch drei Lebensläufe von Neurologen während der NS-Zeit vorstellen und die Entwicklung der deutschsprachigen Neurologie unter dem NS-Regime kritisch einordnen.

Ferner wird es ein gemeinsames Treffen deutscher und französischer Neurologen geben, mit dem Ziel, existierende Synergien zu verbreitern. 2015 fand ein solcher Gedankenaustausch in Paris statt - nun gibt es die Gegeneinladung nach Mannheim.

Ich freue mich auf einen anregenden Wissensund Erfahrungsaustausch mit Ihnen in Mannheim!

Herzliche kollegiale Grüße
Ihr

\section{Stefan Schwab}

Prof. Dr. med. Dr. h.c. Stefan Schwab

DGN-Kongresspräsident,

Ärztlicher Direktor der Neurologie am

Universitätsklinikum Erlangen

Schwabachanlage 6, 91054 Erlangen

E-Mail: direktion-neurologie@uk-erlangen.de 